\title{
Exosome-encapsulated microRNA-23b as a minimally invasive liquid biomarker for the prediction of recurrence and prognosis of gastric cancer patients in each tumor stage
}

\author{
YOSHIMASA KUMATA, HISAE IINUMA, YUSUKE SUZUKI, DAISUKE TSUKAHARA, \\ HIRONORI MIDORIKAWA, YUICHI IGARASHI, NARUYOSHI SOEDA, TAKASHI KIYOKAWA, \\ MASAHIRO HORIKAWA and RYOJI FUKUSHIMA
}

Department of Surgery, Teikyo University School of Medicine, Itabashi, Tokyo 173-0003, Japan

Received October 20, 2017; Accepted April 17, 2018

DOI: 10.3892/or.2018.6418

\begin{abstract}
Recently, exosome-encapsulated microRNAs (miRNAs) have been attracting attention as stable and minimally invasive biomarkers in cancer patients. The aim of the present study was to clarify the value of plasma exosomal microRNA-23b (miR-23b) as a diagnostic and prognostic biomarker in gastric cancer (GC) patients at each tumor stage. We first selected recurrence specific exosomal miRNA by miRNA microarray from $6 \mathrm{GC}$ patients (stage I) with or without recurrence, and 3 healthy volunteers. In this analysis, miR-23b demonstrated the most significant change. Subsequently, we validated the usefulness of miR-23b as a biomarker using the plasma exosome samples collected from $232 \mathrm{GC}$ patients and 20 healthy volunteers. miR-23b levels were evaluated by Taqman microRNA assays. Exosomal miR-23b levels of GC patients were significantly lower than those of the healthy controls. A significant association was revealed between the plasma exosomal miR-23b levels and the expression of miR-23b in primary tumor tissues. Concerning the pathological condition, miR-23b demonstrated a significant association with tumor size, depth of invasion, liver metastasis and TNM stage. The overall survival (OS) rates of low-miR-23b patients were significantly worse than those of high-miR-23b patients at stage I, II, III and IV. The disease-free survival (DFS) rates of low exosomal miR-23b patients were significantly worse than those of high-miR-23b patients at stage I, II and III. Cox multivariate analysis indicated that exosomal miR-23b was an independent prognostic factor for OS and DFS at each tumor stage. Our results revealed that exosomal miR-23b has potential
\end{abstract}

Correspondence to: Dr Hisae Iinuma, Department of Surgery, Teikyo University School of Medicine, 2-11-1 Kaga, Itabashi, Tokyo 173-0003, Japan

E-mail: iinuma@med.teikyo-u.ac.jp

Abbreviations: GC, gastric cancer; miRNA, microRNA; qRT-PCR, quantitative real-time reverse transcription-PCR

Key words: gastric cancer, liquid biomarker, exosome, microRNA-23b, prognosis as minimally invasive predictive biomarker for the recurrence and prognosis of GC in patients at all stages.

\section{Introduction}

Gastric cancer (GC) is one of the most prevalent cancers in Japan and other East Asian countries (1). Although the 5-year survival rate of GC patients with early stage in Japan is $97 \%$, the recurrence rate after surgery in GC patients with advanced stage is also high. In particular, the 5-year survival rate at stage IV is around $10 \%$. In order to improve the prognosis, it is important to clarify the biomarkers for the screening of GC patients in the high-risk group of recurrence.

MicroRNAs (miRNAs) have been shown to be one of the potential biomarkers for tumor diagnosis and prediction of prognosis in various types of cancer $(2,3)$. They are small non-coding 23-35 nucleotide molecules, which post-transcriptionally regulate the production of proteins from their messenger RNAs (mRNAs) (3). miRNAs play an important role in the process of cell proliferation, differentiation, apoptosis and metastasis (2). It has been reported that miRNAs are abnormally expressed in cancers and influence the initiation and progression of cancer cells as oncogenes or tumor-suppressor genes (4). In addition, miRNAs can be detected not only in tissues, but also in body fluids such as plasma, serum, urine, saliva and lactation milk. Many studies have focused on cancer-derived miRNAs in the circulatory system of cancer patients $(5,6)$. These studies indicated that plasma miRNAs may act as minimally invasive biomarkers for the diagnosis and prognosis of GC patients (5-8).

Other studies have confirmed the existence of miRNAs in a stable form within plasma/serum exosome. The exosomes, which are extremely small at $40-150 \mathrm{~nm}$, originate from the luminal membranes of multivesicular bodies $(9,10)$. Released by the process of fusion with the cell membranes of multivesicular bodies, these exosomes contain protein and selectively packaged RNA, such as miRNA, and have the ability to transfer these components to other cells $(11,12)$. Cancer patients often exhibit high concentrations of exosomes, and if the exosomes contain intact miRNA, they have potential as effective predictive and prognostic biomarkers (12-15). At 
present, we have already reported that exosome miR-21 is a useful biomarker for predicting the recurrence and prognosis of lung and colorectal cancer $(16,17)$. However, few published studies have investigated the association between the plasma exosomal miRNA expression and the prognosis of GC patients at each tumor stage.

In the present study, we aimed to demonstrate the potential of exosome-encapsulated miRNAs as predictive biomarkers for recurrence and prognosis in GC patients at each tumor stage.

\section{Patients and methods}

Study design. We selected recurrence specific exosomal miRNA by microRNA micro-array analysis using the plasma exosomes collected from stage I GC patients who had relapsed after surgery $(n=3)$, stage I GC patients who had not relapsed after surgery $(n=3)$ and healthy controls $(n=3)$. Subsequently, we validated the selected miRNA using the plasma exosomes collected from another 232 GC patients and 20 healthy volunteers. The patients were studied between November 2006 and December 2013 at Teikyo University Hospital. The cancer stage was determined according to the TNM classification (UICC). In the present study, 74 cases with stage I, 47 cases with stage II, 79 cases with stage III and 32 cases with stage IV GC were included. The median follow-up period was 3.8 years (range, 0.4-10.6 years). The samples were collected before the start of the treatment. The patients were treated with standard treatment for GC patients. The study protocol conformed to the guidelines of the ethics committee of the Teikyo University, and was approved by the review board of the Teikyo University (09-081-3). Written informed consent was obtained from the all patients.

Patients follow-up. Post-operative follow-up was performed according to the guidelines published by the Japanese Gastric Cancer Association (18). Confirmation of recurrence was required to evaluate imaging or pathological diagnosis. Testing of the tumor markers (CEA and CA19-9), combined with a general physical examination, were conducted every 3 months for 3 years and then every 6 months for 5 years. Following surgery, computed tomography was conducted once every 6 months for 5 years and then every 6 or 12 months for up to 10 years. Gastroscopy was conducted annually for a period of 5 years after surgery.

Purification of exosomes from plasma and recognition by transmission electron microscopy. Plasma $(1 \mathrm{ml})$ separated from blood was used for microarray analysis and quantitative real-time reverse transcription-PCR (qRT-PCR). The exosomes were separated by ultracentrifugation $(15,000 \mathrm{x} \mathrm{g}$ for $70 \mathrm{~min}$ ) from the plasma, and the isolated exosomes were recognized by transmission electron microscopy using the electron microscope H-7600 (Hitachi High-Technologies Corp., Tokyo, Japan) as previously described (17).

Total RNA isolation from exosomes and tissues. Total RNAs (including the miRNA) of exosomes were isolated using the miRNeasy Serum/Plasma kit (Qiagen, Venlo, The Netherlands) and total RNAs (including the miRNA) of tissues were extracted using the miRNeasy Mini kit (Qiagen). Subsequent extraction and cartridge work was performed according to the manufacturer's protocol as previously described (17). The quality of extracted RNA was assessed using an Agilent 2100 Bioanalyzer (Agilent Technologies, Santa Clara, CA, USA).

miRNA microarray analysis. Examination of the exosomal miRNA expression profiles was conducted with a 3D-Gene Human miRNA Oligo chip ver.20 (Toray Industries Inc. Tokyo, Japan). In total, 2,578 genes were mounted in this chip. A 3D-Gene scanner (Toray) was used to scan and analyze the fluorescence signals. All procedures were conducted according to the manufacturer's protocol. The raw data for each spot were normalized to the mean intensity of background signals determined by all blank signal intensities at $95 \%$ confidence intervals. Effective assessements were considered when the signal intensity of both duplicate spots was $>2$ SD of the background signal intensity.

Quantitative real time-PCR( $q R T-P C R)$ formiRNAs ofexosomes and tissues. By using qRT-PCR, the miRNA expression from the plasma exosomes and tissues was examined. Synthesis of cDNA of total RNA isolated from exosomes was conducted using TaqMan microRNA primers specific for miR-23b and miR-16a (Thermo Fisher Scientific Inc., Waltham, MA, USA), and TaqMan Micro-RNA Reverse Transcription kit (Thermo Fisher Scientific). Since previous research had reported that miR-16a was a reliable endogenous control for miRNA analysis by qRT-PCR in human plasma samples, we decided to use it as an internal control. TaqMan microRNA primers specific for miR-23b and RNU6B and TaqMan Micro-RNA Reverse Transcription kit (Thermo Fisher Scientific) were employed to synthesize the cDNA of total RNA isolated from tissues. RNU6B was selected as the internal control of tissue samples. qRT-PCR was performed using TaqMan Universal PCR Master Mix (Thermo Fisher Scientific) and LightCycler-480 (Roche Applied Science, Basel, Switzerland) following the manufacturer's protocol. Each sample was analyzed in duplicate. Relative quantification of miRNA expression was calculated using the $2^{-\Delta \Delta C T}$ method as previously described (17).

Statistical analysis. The data were expressed as the mean \pm standard deviation (SD). The cut-off value was set at 0.78 , which is the median of miR-23b. The relationship between the microRNA expression and clinicopathological factors was analyzed using the Student's t-test, the Chi-square test and ANOVA. Using the Kaplan-Meier survival curves, overall survival (OS) and disease-free survival (DFS) curves were analyzed, and the differences were estimated using log-rank tests. Cox proportional hazard model was used to estimate univariate and multivariate hazard ratios for OS and DFS. Multivariate analysis was performed for factors that showed significance in univariate analysis. All P-values are two-sided, and $\mathrm{P}<0.05$ was considered to indicate a statistically significant difference. Statistical analyses were performed using the JMP 9.0 software (SAS Institute, Inc., Cary, NC, USA).

\section{Results}

Exosome electron microscopic image. To confirm the exosomes, we examined the ultracentrifugation samples from the plasma of GC patients by transmission electron micros- 
Table I. Background of 6 GC patients and 3 healthy volunteers subjected to microRNA array analysis.

A, GC patients

\begin{tabular}{|c|c|c|c|c|c|c|}
\hline Case no. & 1 & 2 & 3 & 4 & 5 & 6 \\
\hline Age/Race & 61/Jpn. & 60/Jpn. & 66/Jpn. & 59/Jpn. & 72/Jpn. & 76/Jpn. \\
\hline Sex & $\mathrm{F}$ & M & M & M & $\mathrm{F}$ & M \\
\hline TNM stage & $\mathrm{I}$ & $\mathrm{I}$ & $\mathrm{I}$ & $\mathrm{I}$ & I & $\mathrm{I}$ \\
\hline Recurrence (location) & - & - & - & + (Liver) & + (Liver) & + Liver) \\
\hline Tumor size $(\mathrm{cm})$ & 2.3 & 6.3 & 5.0 & 4.5 & 6.7 & 5.5 \\
\hline Differentiation & Mod & Mod & Por & Mod & Mod & Por \\
\hline Tumor differentiation & $\mathrm{T} 2$ & $\mathrm{~T} 2$ & $\mathrm{~T} 2$ & $\mathrm{~T} 2$ & $\mathrm{~T} 2$ & $\mathrm{~T} 2$ \\
\hline Lymph node metastasis & n (-) & n (-) & n (-) & n (-) & n (-) & n (-) \\
\hline Clinical outcome & Survival & Survival & Survival & Death & Death & Death \\
\hline
\end{tabular}

B, Healthy volunteers

\begin{tabular}{lccc}
\hline No. & 1 & 2 & 3 \\
\hline Age/race & $71 / \mathrm{Jpn}$. & $62 / \mathrm{Jpn}$. & $63 / \mathrm{Jpn}$. \\
Sex & $\mathrm{M}$ & $\mathrm{F}$ & $\mathrm{M}$
\end{tabular}

M, male; F, female; Jpn, Japanese; Por, poorly differentiated; Mod, moderately differentiated; GC, gastric cancer.

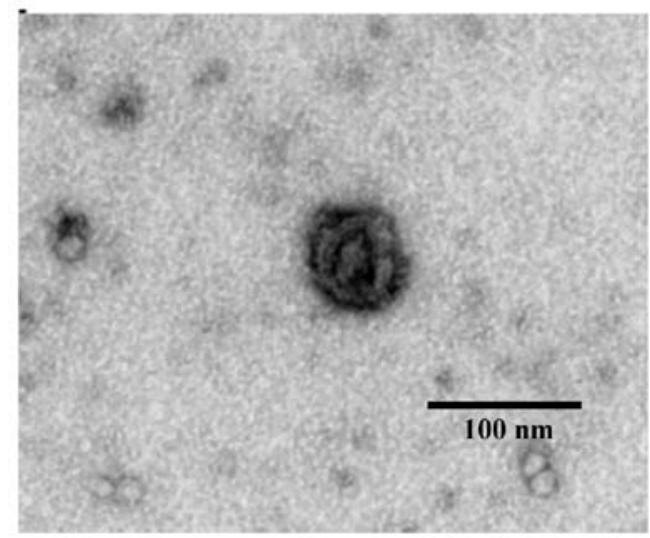

Figure 1. Exosome images captured by transmission electron microscope.

copy. In this sample, we captured images of round micro vesicles that had diameters of about 50-100 nm (Fig. 1).

Exosomal miRNA array analysis of GC patients. To reveal the recurrence-predictor exosomal miRNA in GC patients, microRNA array analysis was employed. In the present study, plasma exosome samples were collected from stage I GC patients who showed recurrence after surgery (recurrence group, $n=3$ ), stage I GC patients who did not show any recurrence after surgery (non-recurrence group, $n=3$ ) and a healthy control group $(n=3)$. The clinical backgrounds of these $6 \mathrm{GC}$ patients and 3 healthy controls used for this analysis are listed in Table I. The recurrence sites of 3 patients were liver. Table II demonstrates the five markedly downregulated and upregulated exosomal miRNAs after comparison of these samples. In these miRNAs, miR-23b (MIMAT0000418) of the recurrence group

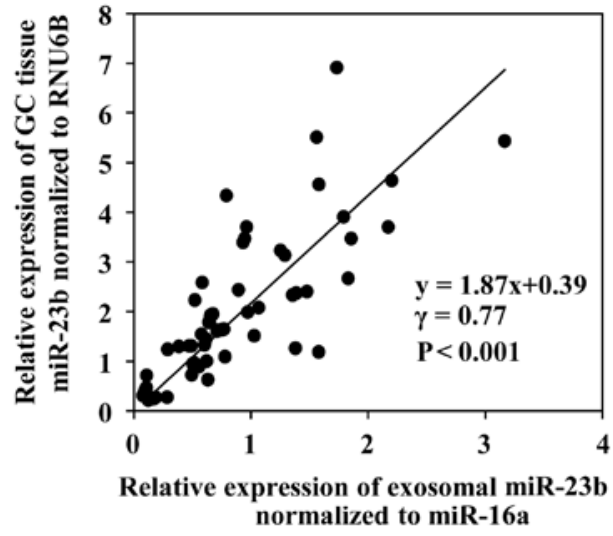

Figure 2. Correlation between miR-23b levels in plasma exosomes and tissues of GC patients. Pair samples (plasma and tissue) from 60 patients with stage I $(n=15)$, stage II $(n=15)$, stage III $(n=15)$ and stage IV $(n=15)$ GC were included in the present study. GC, gastric cancer. $\mathrm{P}<0.001$.

displayed the most marked change compared to that of the healthy control and non-recurrence group. In the data for the upregulated miRNAs, fold changes were at lower levels than those of the downregulated miRNAs. These results led us to select miR-23b as a potential predictive marker in GC patients.

Expression of miR-23b in the GC tissues and plasma exosomes. Expression of miR-23b was assessed by qRT-PCR in plasma exosomal samples and primary tissues collected from GC patients. First, we examined the correlation between exosomal miR-23b levels and miR-23b expression in primary tumor tissues in the same patients. Sixty patients with stage I (15 cases), stage II (15 cases), stage III (15 cases) or stage IV (15 cases) GC were subjected in this analysis. As displayed in Fig. 2, a 
Table II. The 5 markedly downregulated and upregulated miRNAs in plasma exosomes of stage I GC patients with recurrence by miRNA array analysis.

Fold-change

\begin{tabular}{lllcc} 
Ranks & microRNA & MiRBase no. & $\begin{array}{c}\text { Recurrent GC vs. } \\
\text { healthy controls }\end{array}$ & $\begin{array}{c}\text { Recurrent GC vs. } \\
\text { non-recurrent GC }\end{array}$ \\
\hline Downregulation & & & & 0.35 \\
1 & miR-23b-3p & MIMAT 0000418 & 0.30 & 0.52 \\
2 & miR-3135b & MIMAT 0018985 & 0.33 & 0.55 \\
3 & miR-6131 & MIMAT 0024615 & 0.35 & 0.60 \\
4 & miR-6850-3p & MIMAT 0027601 & 0.38 & 0.62 \\
5 & miR-187-5p & MIMAT 0004561 & 0.49 & 2.15 \\
Upregulation & & & & 2.13 \\
1 & miR-21-5p & MIMAT 0000076 & 2.58 & 2.27 \\
2 & miR-106a-5p & MIMAT 0000103 & 2.54 & 2.17 \\
3 & miR-221-3p & MIMAT 0000278 & 2.53 & 2.12 \\
4 & miR-223-3p & MIMAT 0000280 & 2.53 & 2.47 \\
5 & miR-6511a-5p & MIMAT 0025478 & & \\
\hline
\end{tabular}

GC, gastric cancer.
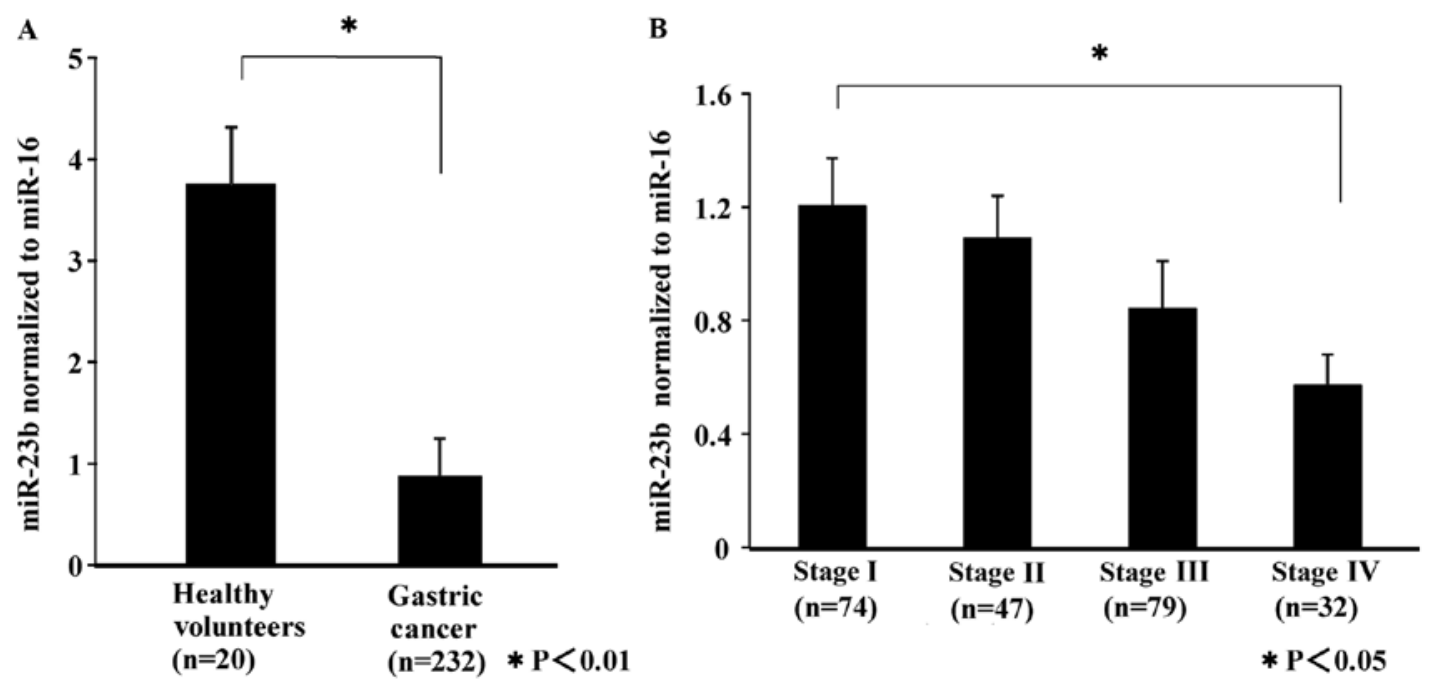

Figure 3. Plasma exosomal miR-23b levels. (A) Exosomal miR-23b levels in healthy controls and GC patients, $\mathrm{P}<0.01$. (B) Exosomal miR-23b levels in GC patients at each tumor stage. Exoxomal miR-23b levels of patients with stage IV GC is significantly lower than that of stage I GC (P<0.05). GC, gastric cancer.

positive significant correlation was demonstrated between them $(\mathrm{P}<0.01)$. Subsequenlty, exosomal miR-23b levels from 232 patients with stage I (74 cases), stage II (47 cases), stage III ( 79 cases) and stage IV ( 32 cases) GC and 20 healthy volunteers were compared. As displayed in Fig. 3A, exosomal miR-23b levels of GC patients were significantly lower than those of the healthy controls $(\mathrm{P}<0.01)$. Furthermore, with the progression of cancer, exosomal miR-23b levels decreased (Fig. 3B). In stage IV patients, the miR-23b levels decreased significantly $(\mathrm{P}<0.05)$.

Association between exosomal miR-23b levels and clinicopathological factors. To evaluate the correlation between the expression of exosomal miR-23b levels and the clinicopatho- logical factors, 232 patients were divided into two groups, in which the expression of exosomal miR-23b levels was either high or low (Table III). The cut-off level was determined as the median of the miR-23a expression levels (0.78). A statistically significant association was observed between the miR-23b and the tumor size, depth of invasion, liver metastasis and TNM stage.

Kaplan-Meier OS and DFS survival curves based on exosomal miR-23b levels. A comparison was made between the Kaplan-Meier OS curves of all patients $(n=232)$ and the DFS curves of patients who had experienced curative surgery $(n=200)$. In all patients, the low miR-23b group exhibited a significantly worse OS than those in the high miR-23b group 
Table III. Relationship between the clinicopathological factors of patients and the expression of miR-23b.

\begin{tabular}{|c|c|c|c|}
\hline Variables & miR-23b low $(\mathrm{n}=100), \mathrm{n}(\%)$ & miR-23b high $(\mathrm{n}=132), \mathrm{n}(\%)$ & P-value \\
\hline \multicolumn{4}{|l|}{ Sex } \\
\hline Male & $74(74.0)$ & $91(68.9)$ & \multirow[t]{2}{*}{0.400} \\
\hline Female & $26(26.0)$ & $41(31.1)$ & \\
\hline \multicolumn{4}{|l|}{ Tumor size (cm) } \\
\hline$<5$ & $36(36.0)$ & $68(51.5)$ & \multirow[t]{2}{*}{0.019} \\
\hline$\geqq 5$ & $64(64.0)$ & $64(48.5)$ & \\
\hline \multicolumn{4}{|l|}{ Differentiation } \\
\hline Well/moderate & $60(60.0)$ & $67(50.8)$ & \multirow[t]{2}{*}{0.161} \\
\hline Poor/other & $40(40.0)$ & $65(49.2)$ & \\
\hline \multicolumn{4}{|c|}{ Depth of invasion } \\
\hline pT1 & $21(21.0)$ & $45(34.1)$ & \multirow[t]{2}{*}{0.029} \\
\hline$\geqq \mathrm{pT} 2$ & $79(79.0)$ & $87(65.9)$ & \\
\hline \multicolumn{4}{|c|}{ Lymphatic invasion } \\
\hline Ly (-) & $35(35.0)$ & $64(48.5)$ & \multirow[t]{2}{*}{0.184} \\
\hline Ly (+) & $65(65.0)$ & $68(51.5)$ & \\
\hline \multicolumn{4}{|l|}{ Venous invasion } \\
\hline $\mathrm{V}(-)$ & $39(39.0)$ & $58(43.9)$ & \multirow[t]{2}{*}{0.450} \\
\hline $\mathrm{V}(+)$ & $61(61.0)$ & $74(56.1)$ & \\
\hline \multicolumn{4}{|c|}{ Lymph node metastasis } \\
\hline $\mathrm{N}(-)$ & $35(35.0)$ & $59(44.7)$ & \multirow[t]{2}{*}{0.136} \\
\hline $\mathrm{N}(+)$ & $65(65.0)$ & $73(55.3)$ & \\
\hline \multicolumn{4}{|l|}{ Liver metastasis } \\
\hline H (-) & $93(93.0)$ & $130(98.5)$ & \multirow[t]{2}{*}{0.032} \\
\hline $\mathrm{H}(+)$ & $7(7.0)$ & $2(1.5)$ & \\
\hline \multicolumn{4}{|c|}{ Peritoneum dissemination } \\
\hline$P(-)$ & $87(87.0)$ & $121(91.7)$ & \multirow[t]{2}{*}{0.248} \\
\hline $\mathrm{P}(+)$ & $13(13.0)$ & $11(8.3)$ & \\
\hline \multicolumn{4}{|c|}{ Distant metastasis } \\
\hline M (-) & $93(93.0)$ & $128(95.5)$ & \multirow[t]{2}{*}{0.282} \\
\hline $\mathrm{M}(+)$ & $7(7.0)$ & $4(4.5)$ & \\
\hline \multicolumn{4}{|l|}{ TNM stage } \\
\hline I & $24(24.0)$ & $50(37.9)$ & \multirow[t]{4}{*}{0.034} \\
\hline II & $22(22.0)$ & 25 (18.9) & \\
\hline III & $34(34.0)$ & $45(34.1)$ & \\
\hline IV & $20(20.0)$ & $12(9.1)$ & \\
\hline
\end{tabular}

(Fig. 4A). In those patients who had undergone curative surgery, the low miR-23b group showed a significantly worse DFS than those in the high miR-23b group (Fig. 4B). An analysis of the data at each stage revealed that, in patients with stage I $(n=74)$, the low miR-23b group showed a significantly worse OS and DFS than those in the high miR-23b group (Fig. 5). In patients with stage II GC $(n=47)$, the low miR-23b group showed a significantly worse OS and DFS than those in the high miR-23b group (Fig. 6). Among stage III GC patients $(n=79)$, the low miR-23b group had a significantly worse OS and DFS compared to the high miR-23b group (Fig. 7). As for GC patients at stage IV $(n=32)$, the low miR-23b group was observed to have significantly worse OS than those in the high miR-23b group (Fig. 8). These data indicated that a low expression of exosomal miR-23b correlated with recurrence and poor prognosis in all stages.

Univariate and multivariate Cox proportional hazard regression analysis for $O S$ and DFS. Univariate and multivariate Cox analysis for OS and DFS in GC patients was examined. Multivariate analysis was performed for variables that showed significance in univariate analysis. Table IV displays the results of univariate and multivariate analysis for the OS of all patients $(n=232)$ and the DFS of those patients who had received curative surgical procedures $(n=200)$. In the multivariate analysis for OS, depth of invasion, lymphatic invasion, 

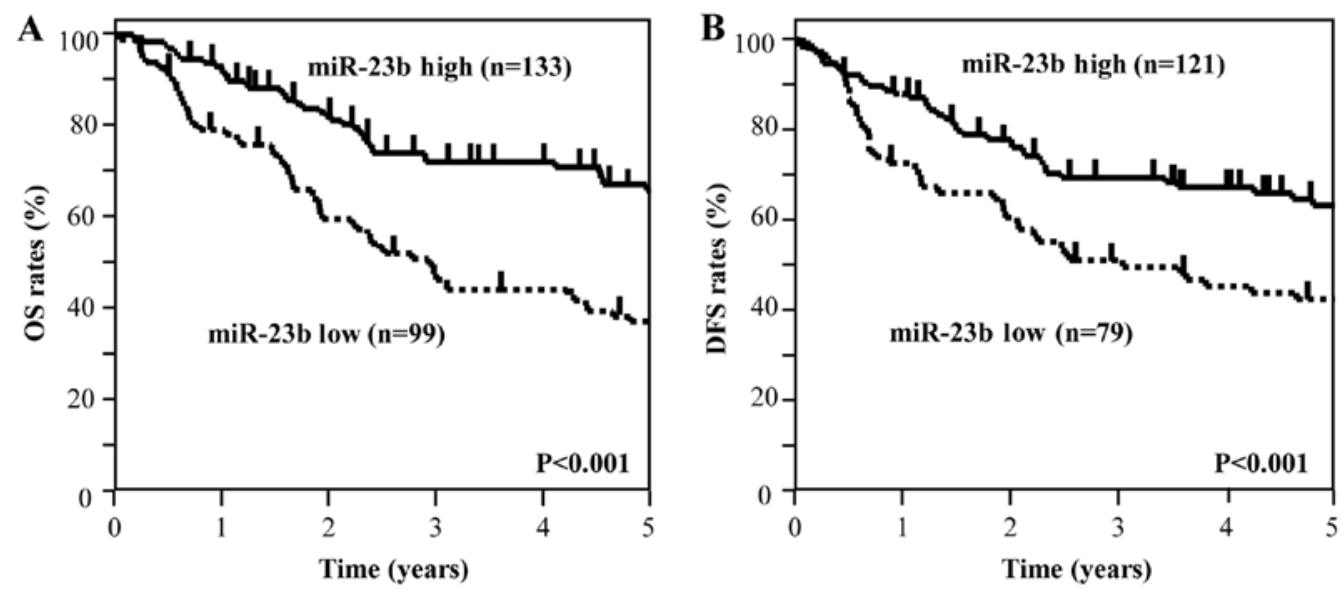

Figure 4. Kaplan-Meier survival curves of overall survival (OS) and disease-free survival rates (DFS) based on exosomal miR-23b levels. (A) Comparison of OS between groups of high and low levels of exosome miR-23b in all GC patients $(\mathrm{n}=232)$. (B) Comparison of DFS between groups with high and low levels of exosome miR-23b in GC patients who had undergone curative surgery $(\mathrm{n}=200)$. OS, overall survival; DFS, disease-free survival.
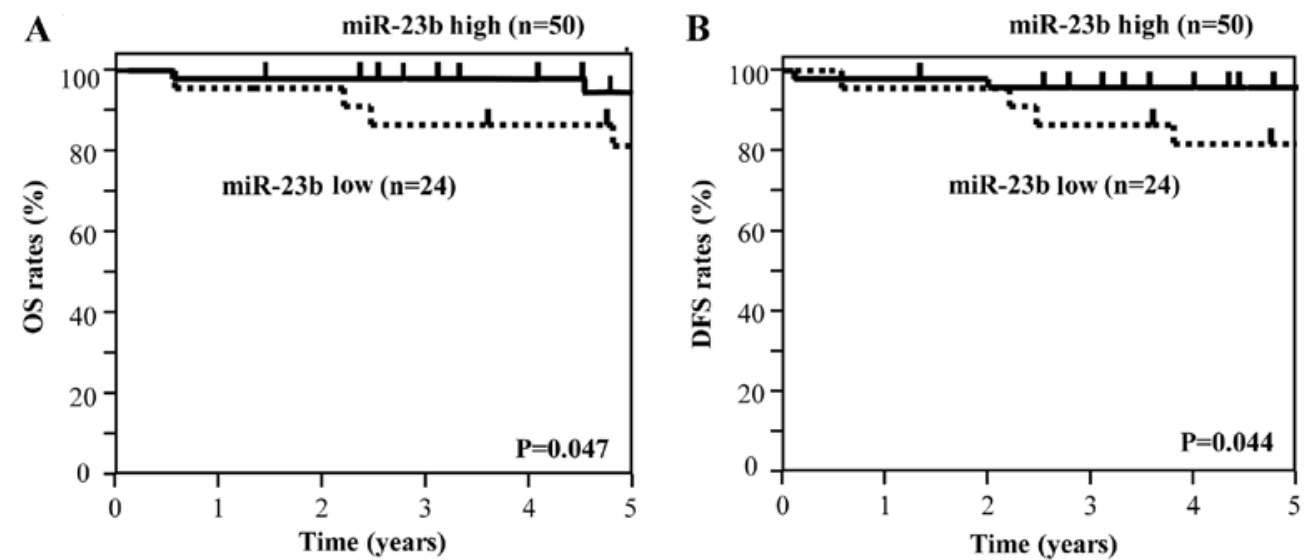

Figure 5. Kaplan-Meier survival curves of OS and DFS based on exosomal miR-23b levels in patients with stage I GC. (A) Comparison of OS between groups with high and low levels of exosome miR-23b in patients with stage I GC (n=74). (B) Comparison of DFS between groups with high and low levels of exosome miR-23b in patients with stage I GC ( $\mathrm{n}=74)$. OS, overall survival; DFS, disease-free survival; GC, gastric cancer.
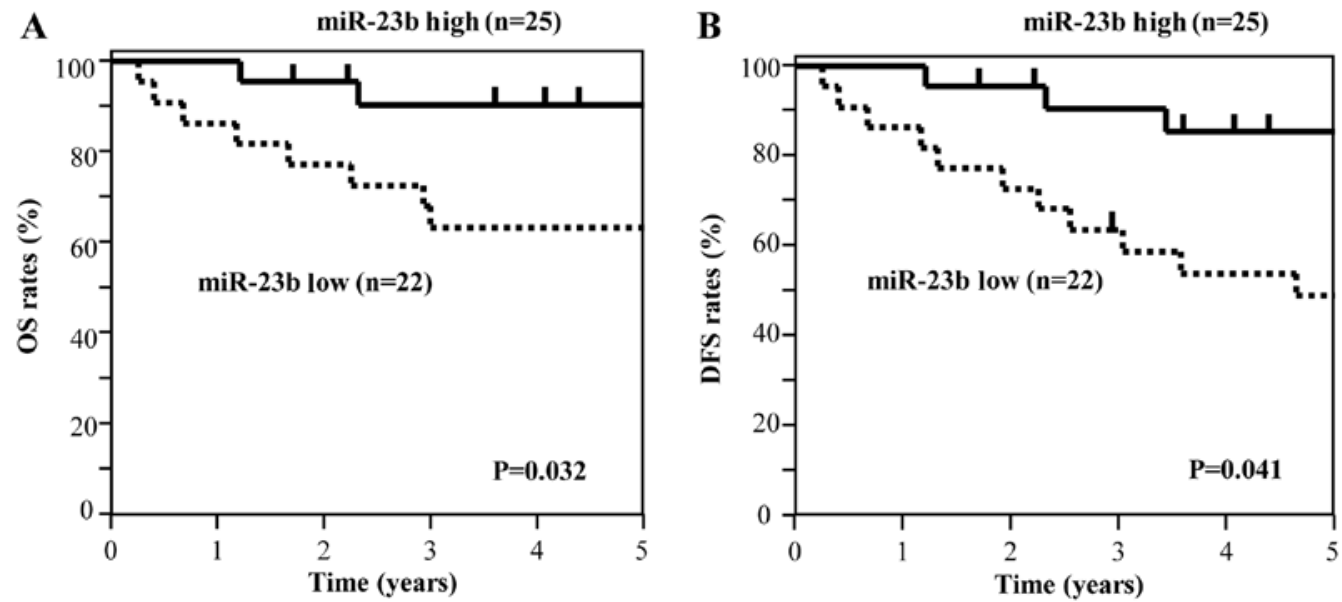

Figure 6. Kaplan-Meier survival curves of OS and DFS based on exosomal miR-23b levels in patients with stage II GC. (A) Comparison of OS between groups with high and low levels of exosome miR-23b in patients with stage II GC $(n=47)$. (B) Comparison of DFS between groups with high and low levels of exosome miR-23b in patients with stage II GC $(\mathrm{n}=47)$. OS, overall survival; DFS, disease-free survival; $\mathrm{GC}$, gastric cancer.

liver metastasis, peritoneum dissemination and exosomal miR-23b showed significance. In the multivariate analysis for
DFS, tumor size, depth of invasion, lymph node metastasis and exosomal miR-23b showed significance for DFS. We then 

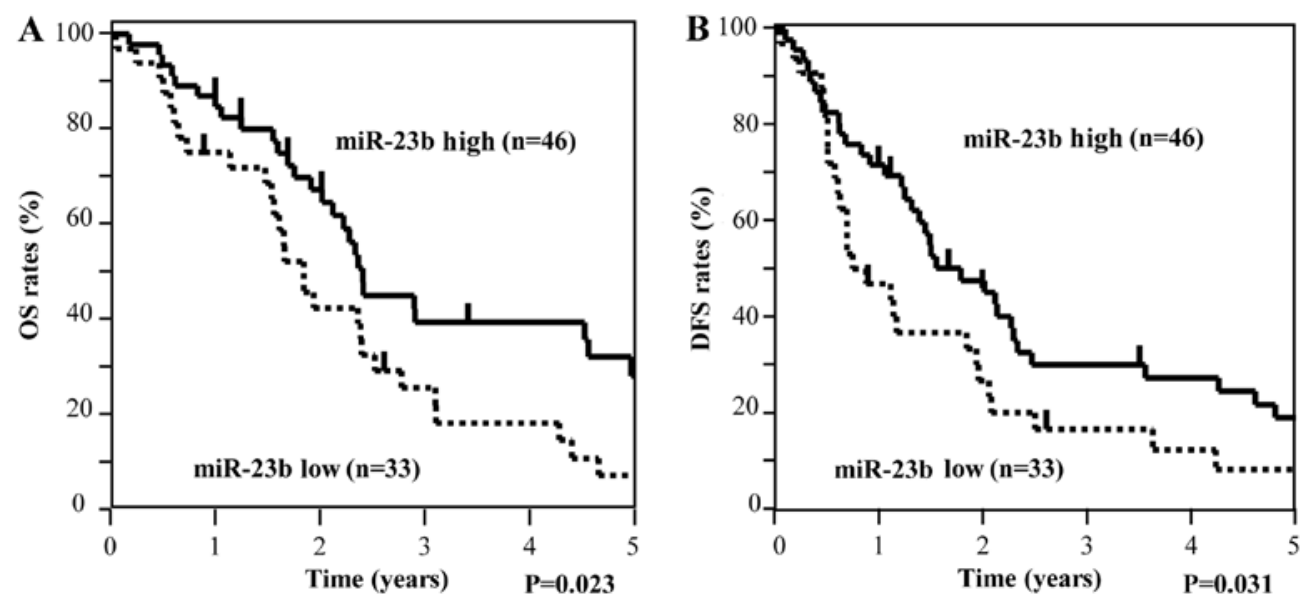

Figure 7. Kaplan-Meier survival curves of OS and DFS based on exosomal miR-23b levels in patients with stage III GC. (A) Comparison of OS between groups with high and low levels of exosome miR-23b in patients with stage III GC $(n=79)$. (B) Comparison of DFS between groups with high and low levels of exosome miR-23b in patients with stage III GC $(\mathrm{n}=79)$. OS, overall survival; DFS, disease-free survival; GC, gastric cancer.

Table IV. Univariate and multivariate Cox analyses for OS in all patients and DFS in patients who had undergone curative surgery.

$\mathrm{A}, \mathrm{OS}$

\begin{tabular}{|c|c|c|c|c|c|c|}
\hline \multirow[b]{2}{*}{ Variables } & \multicolumn{3}{|c|}{ Univariate analysis } & \multicolumn{3}{|c|}{ Multivariate analysis } \\
\hline & $\mathrm{RC}$ & Hazard ratio $(95 \% \mathrm{CI})$ & P-value & $\mathrm{RC}$ & Hazard ratio $(95 \% \mathrm{CI})$ & P-value \\
\hline Tumor size & 1.06 & $2.73(1.79-4.25)$ & $<0.001$ & 0.447 & $1.56(0.99-2.52)$ & 0.065 \\
\hline \multicolumn{7}{|l|}{ Depth } \\
\hline of invasion & 2.69 & $7.74(6.16-8.22)$ & $<0.001$ & 1.833 & $6.25(2.07-9.14)$ & 0.001 \\
\hline Lymph node metastasis & 1.62 & $5.09(3.12-8.17)$ & $<0.001$ & 0.284 & $1.32(0.72-2.53)$ & 0.362 \\
\hline Lymphatic invasion & 1.36 & $3.89(2.45-6.47)$ & $<0.001$ & 0.258 & $2.16(1.20-3.99)$ & 0.010 \\
\hline Venous invasion & 1.17 & $3.22(2.07-5.16)$ & $<0.001$ & 0.463 & $1.58(0.94-2.77)$ & 0.080 \\
\hline Histological type & -0.13 & $0.87(0.58-1.29)$ & 0.513 & & & \\
\hline Liver metastasis & 1.78 & $5.94(2.75-11.35)$ & $<0.001$ & 1.667 & $5.29(1.92-12.42)$ & 0.003 \\
\hline Peritoneum dissemination & 1.18 & $3.27(1.92-5.33)$ & $<0.001$ & 1.224 & $3.40(1.80-6.07)$ & 0.001 \\
\hline $\operatorname{miR}-23 b$ & -0.83 & $0.45(0.29-0.64)$ & $<0.001$ & -0.556 & $0.57(0.37-0.78)$ & 0.011 \\
\hline
\end{tabular}

$\mathrm{B}, \mathrm{DFS}$

\begin{tabular}{|c|c|c|c|c|c|c|}
\hline \multirow[b]{2}{*}{ Variables } & \multicolumn{3}{|c|}{ Univariate analysis } & \multicolumn{3}{|c|}{ Multivariate analysis } \\
\hline & $\mathrm{RC}$ & Hazard ratio $(95 \% \mathrm{CI})$ & P-value & $\mathrm{RC}$ & Hazard ratio $(95 \% \mathrm{CI})$ & P-value \\
\hline Tumor size & 1.12 & $3.38(2.14-5.49)$ & $<0.001$ & 0.557 & $1.74(1.07-2.90)$ & 0.024 \\
\hline \multicolumn{7}{|l|}{ Depth } \\
\hline of invasion & 2.76 & $5.79(6.57-11.78)$ & $<0.001$ & 2.046 & $7.73(2.52-13.87)$ & 0.001 \\
\hline Lymph node metastasis & 1.89 & $6.67(3.88-12.34)$ & $<0.001$ & 0.671 & $1.95(1.03-3.94)$ & 0.039 \\
\hline Lymphatic invasion & 1.45 & $4.24(2.57-7.36)$ & 0.001 & 0.399 & $1.49(0.86-2.69)$ & 0.154 \\
\hline Venous invasion & 1.59 & $4.91(2.92-8.78)$ & 0.001 & 0.474 & $1.60(0.92-2.97)$ & 0.096 \\
\hline Histological type & 0.12 & $1.13(0.74-1.72)$ & 0.565 & & & \\
\hline $\operatorname{miR}-23 b$ & -0.48 & $0.61(0.40-0.75)$ & 0.023 & -0.43 & $0.64(0.41-0.91)$ & 0.041 \\
\hline
\end{tabular}

RC, regression coefficient; OS, overall survival; DFS, disease-free survival.

considered the prognostic value of these factors at each stage of tumor development. In the multivariate analysis of patients with stage I GC, depth of invasion and exosomal miR-23b demonstrated significance for both OS and DFS (Table V). 
Table V. Univariate and multivariate Cox analyses for OS and DFS in patients with stage I GC.

$\mathrm{A}, \mathrm{OS}$

\begin{tabular}{|c|c|c|c|c|c|c|}
\hline \multirow[b]{2}{*}{ Variables } & \multicolumn{3}{|c|}{ Univariate analysis } & \multicolumn{3}{|c|}{ Multivariate analysis } \\
\hline & $\mathrm{RC}$ & $\mathrm{HR}(95 \% \mathrm{CI})$ & P-value & $\mathrm{RC}$ & HR $(95 \%$ CI $)$ & P-value \\
\hline Tumor size & -0.01 & $0.99(0.14-4.62)$ & 0.994 & & & \\
\hline Lymph-node metastasis & 0.71 & $2.04(0.11-12.13)$ & 0.547 & & & \\
\hline Lymphatic invasion & 0.72 & $2.05(0.29-9.52)$ & 0.418 & & & \\
\hline Vascular invasion & 1.37 & $3.92(0.77-17.86)$ & 0.094 & & & \\
\hline Histological type & 0.24 & $1.27(0.28-6.46)$ & 0.752 & & & \\
\hline Depth of invasion & 2.03 & $7.63(1.68-18.79)$ & 0.010 & 2.00 & $7.41(1.63-17.75)$ & 0.021 \\
\hline $\operatorname{miR}-23 b$ & -1.53 & $0.22(0.02-0.87)$ & 0.032 & -1.49 & $0.22(0.04-0.95)$ & 0.043 \\
\hline
\end{tabular}

B, DFS

\begin{tabular}{|c|c|c|c|c|c|c|}
\hline \multirow[b]{2}{*}{ Variables } & \multicolumn{3}{|c|}{ Univariate analysis } & \multicolumn{3}{|c|}{ Multivariate analysis } \\
\hline & $\mathrm{RC}$ & HR $(95 \%$ CI $)$ & P-value & $\mathrm{RC}$ & HR $(95 \%$ CI $)$ & P-value \\
\hline Tumor size & 0.01 & $1.01(0.15-4.71)$ & 0.987 & & & \\
\hline Lymph-node metastasis & 0.82 & $2.26(0.12-13.30)$ & 0.493 & & & \\
\hline Lymphatic invasion & 0.80 & $2.230 .32-10.35)$ & 0.369 & & & \\
\hline Vascular invasion & 1.38 & $3.97(0.78-18.08)$ & 0.091 & & & \\
\hline Histological type & 0.25 & $1.28(0.28-6.50)$ & 0.746 & & & \\
\hline Depth of invasion & 2.20 & $8.98(1.98-15.62)$ & 0.016 & 2.15 & $8.57(1.89-13.57)$ & 0.027 \\
\hline $\operatorname{miR}-23 b$ & -1.63 & $0.20(0.03-0.81)$ & 0.037 & -1.58 & $0.21(0.03-0.91)$ & 0.044 \\
\hline
\end{tabular}

RC, regression coefficient; GC, gastric cancer; OS, overall survival; DFS, disease-free survival.

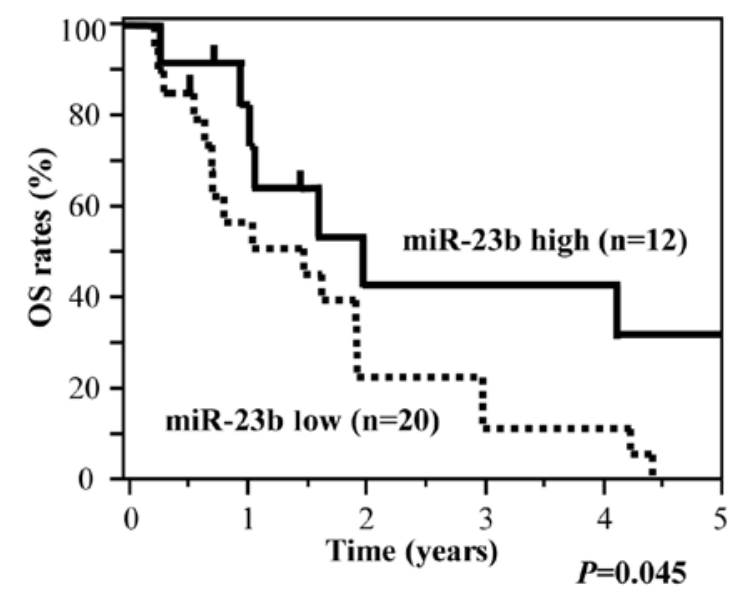

Figure 8. Kaplan-Meier survival curves of OS based on exosomal miR-23b levels in patients with stage IV GC $(\mathrm{n}=32)$. OS, overall survival; $\mathrm{GC}$, gastric cancer.

In the multivariate analysis of patients with stage II GC, exosomal miR-23b showed significance for OS and DFS (Table VI). In the multivariate analysis of patients with stage III GC, exosomal miR-23b showed significance for OS and DFS (Table VII). The multivariate analysis at stage IV GC patients indicated that exosomal miR-23b showed significance for OS (Table VIII). These results led us to believe that plasma exosomal miR-23b levels were an independent prognostic factor in GC patients of all four stages of tumor development.

\section{Discussion}

In the present study, we demonstrated that plasma exosomal miR-23b offers a great potential as a minimally invasive predictive biomarker for recurrence and prognosis in $\mathrm{GC}$ patients. Low expression of exosomal miR-23b indicated a poor prognosis for OS in GC patients at stage I, II, III and IV as well as for DFS in GC patients at stage I, II and III.

Recently, many studies have revealed that miRNAs are stable in the exosomes and show promise as biomarkers. They are minimally invasive in several types of cancers, including GC $(9,10)$. It is known that plasma exosome plays an important role in cell-to-cell signaling (12-14). In this study, firstly we selected a recurrence-predictor exosomal miRNA using the miRNA microarray. miR-23b expressed the lowest downregulation in stage I GC patients who showed recurrence after surgery (recurrence group) compared with that of stage I GC patients who did not show recurrence after surgery (non-recurrence group) and healthy controls. We also examined the miRNA which demonstrated upregulation in this miRNA microarray analysis. However, differences between the recurrence, non-recurrence and healthy control group were small. 
Table VI. Univariate and multivariate Cox analyses for OS and DFS in patients with stage II GC.

$\mathrm{A}, \mathrm{OS}$

\begin{tabular}{|c|c|c|c|c|c|c|}
\hline \multirow[b]{2}{*}{ Variables } & \multicolumn{3}{|c|}{ Univariate analysis } & \multicolumn{3}{|c|}{ Multivariate analysis } \\
\hline & $\mathrm{RC}$ & $\mathrm{HR}(95 \% \mathrm{CI})$ & P-value & $\mathrm{RC}$ & $\operatorname{HR}(95 \% \mathrm{CI})$ & P-value \\
\hline Tumor size & 1.24 & $3.44(1.17-11.40)$ & 0.029 & 1.07 & $2.90(0.97-9.75)$ & 0.061 \\
\hline Lymph-node metastasis & -0.53 & $0.59(0.20-1.74)$ & 0.333 & & & \\
\hline Lymphatic invasion & -0.15 & $0.86(0.29-3.15)$ & 0.806 & & & \\
\hline Vascular invasion & 1.25 & $3.49(0.68-63.54)$ & 0.153 & & & \\
\hline Histological type & 0.78 & $2.19(0.75-6.78)$ & 0.152 & & & \\
\hline Depth of invasion & 2.04 & $8.77(2.87-9.76)$ & 0.469 & & & \\
\hline $\operatorname{miR}-23 b$ & -1.14 & $0.32(0.07-0.78)$ & 0.025 & -1.25 & $0.39(0.09-0.88)$ & 0.042 \\
\hline
\end{tabular}

B, DFS

\begin{tabular}{|c|c|c|c|c|c|c|}
\hline \multirow[b]{2}{*}{ Variables } & \multicolumn{3}{|c|}{ Univariate analysis } & \multicolumn{3}{|c|}{ Multivariate analysis } \\
\hline & $\mathrm{RC}$ & HR $(95 \%$ CI $)$ & P-value & $\mathrm{RC}$ & HR $(95 \%$ CI $)$ & P-value \\
\hline Tumor size & 1.21 & $3.37(1.15-11.08)$ & 0.027 & 1.07 & $2.91(0.98-9.63)$ & 0.057 \\
\hline Lymph-node metastasis & -0.39 & $0.67(0.23-1.97)$ & 0.464 & & & \\
\hline Lymphatic invasion & -0.13 & $0.88(0.29-3.22)$ & 0.833 & & & \\
\hline Vascular invasion & 1.40 & $4.04(0.80-73.30)$ & 0.100 & & & \\
\hline Histological type & 0.79 & $2.20(0.76-6.74)$ & 0.144 & & & \\
\hline Depth of invasion & 2.05 & $8.07(2.31-8.87)$ & 0.393 & & & \\
\hline $\operatorname{miR}-23 b$ & -1.37 & $0.26(0.06-0.72)$ & 0.020 & -1.23 & $0.29(0.07-0.91)$ & 0.040 \\
\hline
\end{tabular}

RC, regression coefficient; GC, gastric cancer; OS, overall survival; DFS, disease-free survival.

Therefore, we selected miR-23b as a predictive biomarker for recurrence of GC patients.

miR-23b is a member of the miR-23b/27b/24 cluster (9q22.32). Functionally, overexpression of miR-23b functions as a tumor suppressor and it has been shown to inhibit migration, proliferation, invasion and tumor growth in various cancers (19-21). Zhuang et al (22) as well as Pellegrino et al (23) have revealed that miR-23b is a tumor-suppressor microRNA, and that low-expression level of miR-23b was associated with metastasis in patients with breast and colon cancer.

In the present study, we revealed that plasma exosomal miR-23b levels of GC patients were significantly lower than those of healthy individuals. These results indicated that miR-23b may be useful in the diagnosis of GC patients. Using tumor tissues, many researchers have demonstrated that the expression of miR-23b wass significantly downregulated in prostate, hepatocellular, bladder and colon cancer $(19,24,25)$. Using the plasma samples (but not exosomes), Kou et al (26) reported that miR-23b was significantly downregulated in colon cancer patients. Although these studies did not use exosomes, the results obtained may support our findings. In addition, our data revealed a significant association between the exosomal miR-23b levels and the expression of miR-23b in primary tumor tissues collected from the same patients. Our results indicated that the tumor tissues may be the source of plasma exosomal miR-23b. Furthermore, we evaluated the relationship between exosomal miR-23b levels and the clinicopathological factors of patients, and revealed that the expression of miR-23b had a significant association with tumor size, depth of invasion, liver metastasis and stage. In the present study, miR-23b was selected by miRNA microarray analyses which were performed between patients without recurrence (non-recurrence) and patients with liver metastasis. Therefore, plasma miR-23b may have shown a significant relationship with 'liver metastasis', not 'peritoneal dissemination' or 'distant metastasis'. Using tissues samples, but not exosomes, Ma et al (27) reported that miR-23b levels were associated with lymph node metastasis, stage and depth of invasion.

The prognostic value of plasma miR-23b levels has been reported in patients with various types of cancer, but the results remained controversial. Kou et al (26) reported that downregulation of miR-23b in plasma was associated with poor prognosis in patients with colorectal cancer. In contrast, Zhuang et al (22) reported that upregulation of plasma miR-23b was associated with a poor prognosis of GC. In this study, the low expression of plasma exosomal miR-23b was significantly associated with poor overall survival and shorter recurrence-free survival in GC patients. The instability of miRNA may be one of the reasons for this controversy. Since miRNAs are preserved in an intact form in exosomes, their stability as biomarkers may be enhanced as a result (15-17). In the present study, we used plasma exosome, and examined its usefulness 
Table VII. Univariate and multivariate analyses of the prognostic factors for OS in patients with stage III GC.

A, OS

\begin{tabular}{|c|c|c|c|c|c|c|}
\hline \multirow[b]{2}{*}{ Variables } & \multicolumn{3}{|c|}{ Univariate analysis } & \multicolumn{3}{|c|}{ Multivariate analysis } \\
\hline & $\mathrm{RC}$ & $\operatorname{HR}(95 \% \mathrm{CI})$ & P-value & $\mathrm{RC}$ & $\mathrm{HR}(95 \% \mathrm{CI})$ & P-value \\
\hline Tumor size & -0.01 & $0.11(0.54-0.91)$ & 0.045 & -2.21 & $0.19(0.72-2.08)$ & 0.113 \\
\hline Lymph-node metastasis & -2.52 & $0.08(0.01-1.52)$ & 0.080 & & & \\
\hline Lymphatic invasion & 0.54 & $1.71(0.79-4.48)$ & 0.186 & & & \\
\hline Vascular invasion & 0.51 & $1.66(0.82-3.83)$ & 0.164 & & & \\
\hline Histological type & -0.26 & $0.77(0.45-1.31)$ & 0.338 & & & \\
\hline Depth of invasion & -1.17 & $0.31(0.06-5.54)$ & 0.331 & & & \\
\hline $\operatorname{miR}-23 b$ & -0.61 & $0.55(0.32-0.73)$ & 0.026 & -0.58 & $0.56(0.33-0.86)$ & 0.037 \\
\hline
\end{tabular}

$\mathrm{B}, \mathrm{DFS}$

\begin{tabular}{|c|c|c|c|c|c|c|}
\hline \multirow[b]{2}{*}{ Variables } & \multicolumn{3}{|c|}{ Univariate analysis } & \multicolumn{3}{|c|}{ Multivariate analysis } \\
\hline & $\mathrm{RC}$ & $\operatorname{HR}(95 \% \mathrm{CI})$ & P-value & $\mathrm{RC}$ & HR $(95 \%$ CI) & P-value \\
\hline Tumor size & -0.07 & $0.93(0.54-1.73)$ & 0.819 & & & \\
\hline Lymph-node metastasis & -1.53 & $0.22(0.04-3.90)$ & 0.227 & & & \\
\hline Lymphatic invasion & 0.56 & $1.74(0.47-0.92)$ & 0.044 & 0.55 & $1.74(0.87-2.01)$ & 0.126 \\
\hline Vascular invasion & 0.35 & $1.42(0.77-2.88)$ & 0.273 & & & \\
\hline Histological type & 0.74 & $3.01(0.62-1.63)$ & 0.985 & & & \\
\hline Depth of invasion & -0.24 & $0.79(0.17-11.98)$ & 0.820 & & & \\
\hline miR-23b & -0.47 & $0.58(0.38-0.81)$ & 0.038 & -0.47 & $0.62(0.41-0.89)$ & 0.044 \\
\hline
\end{tabular}

RC, regression coefficient; GC, gastric cancer; OS, overall survival; DFS, disease-free survival.

Table VIII. Univariate and multivariate Cox analyses for OS in patients with stage IV GC.

\begin{tabular}{|c|c|c|c|c|c|c|}
\hline \multirow[b]{2}{*}{ Variables } & \multicolumn{3}{|c|}{ Univariate analysis } & \multicolumn{3}{|c|}{ Multivariate analysis } \\
\hline & $\mathrm{RC}$ & HR $(95 \% \mathrm{CI})$ & P-value & $\mathrm{RC}$ & HR $(95 \% \mathrm{CI})$ & P-value \\
\hline Tumor size & -0.29 & $0.75(0.32-1.94)$ & 0.524 & & & \\
\hline Lymph-node metastasis & 1.31 & $3.71(1.23-16.22)$ & 0.038 & 0.35 & $1.42(0.27-8.30)$ & 0.679 \\
\hline Lymphatic invasion & 1.31 & $3.69(1.47-11.25)$ & 0.044 & 0.95 & $2.58(0.81-10.98)$ & 0.115 \\
\hline Vascular invasion & 0.72 & $2.06(0.93-4.70)$ & 0.075 & & & \\
\hline Histological type & 0.26 & $1.30(0.50-3.04)$ & 0.566 & & & \\
\hline Depth of invasion & -0.21 & $0.71(0.27-9.78)$ & 0.780 & & & \\
\hline Peritoneum dissemination & -0.54 & $0.58(0.26-1.43)$ & 0.225 & & & \\
\hline Liver metastasis & 0.68 & $1.98(0.83-4.44)$ & 0.118 & & & \\
\hline $\operatorname{miR}-23 b$ & -0.88 & $0.42(0.16-0.87)$ & 0.030 & -0.38 & $0.68(0.24-0.97)$ & 0.042 \\
\hline
\end{tabular}

RC, regression coefficient; GC, gastric cancer; OS, overall survival; DFS, disease-free survival.

as predictive biomarker for recurrence and prognosis of GC patients at each tumor stage. Our results demonstrated that low expression of exosomal miR-23b was significantly associated with poor OS and shorter DFS in GC patients with stage I, II, III and IV. Furthermore, we found that exosomal miR-23b was a significant independent prognostic factor for OS and DFS in GC patients with stage I, II and III and for OS in patients with stage IV. To the best of our knowledge, no previous study has clarified the prognostic value of exosomal miR-23b as a biomarker in patients with GC at each tumor stage. 
The current standard treatment of GC differs according to stage. In Japan, the standard treatment for stage I is endoscopic submucosal dissection or laparoscopic gastrectomy. For patients with stage II and III (except SS/N0 patients), TS-1 is administrated for one year after surgery $(28,29)$. Aggressive postoperative adjuvant chemotherapy, in the form of the administration of capecitabine plus oxaliplatin, is performed in the first half year after surgery for patients with stage III GC (30). However, recurrent cases exist in patients with stage I who underwent curative surgery and in patients with stage II and III who completed postoperative adjuvant chemotherapy. In order to improve prognosis, it is important to clarify high-risk cases of recurrence at each tumor stage. In our study, we revealed that exosomal miR-23b was useful for the selection of GC patients at stage I, II and III who are at high risk of recurrence.

One of the limitations of our study is that it was a retrospective study. Therefore, a larger prospective study is required to clarify the value of exosomal miR-23b. In addition, the target gene of miR23b was not examined in our study. Previous studies have reported Pyk2, Ywhaz, ATG12 and HMGB2 as target genes for miR-23b $(19,20,31,32)$. We are planning to examine these issues in our next study.

In summary, this study has indicated that exosomal miR-23b is a promising, minimally invasive biomarker for the diagnosis, prediction of recurrence and prognosis of patients with GC. Therefore, further development of this exosomal microRNA is expected.

\section{Acknowledgements}

We thank Miss J Tamura for her excellent technical assistance and all the members of the upper gastrointestinal group for their clinical suggestions.

\section{Funding}

The present study was supported by a JSPS KAKENHI (grant nos. JP15K10150 and 17K10608).

\section{Availability of data and materials}

All data generated or analyzed during this study are included in this published article.

\section{Authors' contributions}

HI conceived and designed the study. YK, HI and RF wrote the manuscript. YK and HI performed the experiment. YK, YS, DT, HM, YI, NS, TK and MH collected the clinical data. $\mathrm{HI}$ and RF reviewed and edited the manuscript. All authors read and approved the manuscript and agree to be accountable for all aspects of the research in ensuring that the accuracy or integrity of any part of the work are appropriately investigated and resolved.

\section{Ethics approval and consent to participate}

The study protocol conformed to the guidelines of the Teikyo University Ethics Committee and was approved by the review board of Teikyo University (approval no. 09-081-3). Written informed consent was obtained from all patients.

\section{Consent for publication}

Written informed consent was obtained from all patients for the publication of their data.

\section{Competing interests}

The authors state that they have no competing interests.

\section{References}

1. Uemura N, Okamoto S, Yamamoto S, Matsumura N, Yamaguchi S, Yamakido M, Taniyama K, Sasaki N and Schlemper RJ: Helicobacter pylori infection and the development of gastric cancer. N Engl J Med 345: 784-789, 2001

2. Grady WM and Tewai M: The next thing in prognostic molecular markers: microRNA signatures of cancer. Gut 59: 706-708, 2010.

3. Yi R, Li Y, Wang FL, Miao G, Qi RM and Zhao YY: MicroRNAs as diagnostic and prognostic biomarkers in colorectal cancer. World J Gastrointest Oncol 8: 330-340, 2016.

4. Tsai MM, Wand CS, Tsai CY, Huang HW, Chi HC, Lin YH, $\mathrm{Lu} \mathrm{PH}$ and Lin KH: Potential diagnostic, prognostic and therapeutic target microRNAs in human gastric cancer. Int J Mol Sci 17: E945, 2016.

5. Wang H, Wang L, Wu Z, Sun R, Jin H, Ma J, Liu L, Ling R, Yi J, Wang L, et al: Three dysregulated microRNAs in serum as novel biomarkers for gastric cancer screening. Med Oncol 31: 298,2014

6. Tsujiura M, Komatsu S, Ichikawa D, Shiozaki A, Konishi H, Takeshita H, Moriumura R, Nagata H, Kawaguchi T, Hirajima S, et al: Circulating miR-18a in plasma contributes to cancer detection and monitoring in patients with gastric cancer. Gastric Cancer 18: 271-279, 2015.

7. Ma GJ, Gu RM, Zhu M, Wen X, Li JT, Zhang YY, Zhang XM and Chen SQ: Plasma post-operative miR-21 expression in the prognosis of gastric cancers. Asian Pac J Cancer Prev 14: 7551-7554, 2013.

8. Gorur A, Balci Fidanci S, Dogruer Unal N, Ayaz L, Akbayir S, Yildirim Yaroglu H, Dirlik M, Serin MS and Tamer L: Determination of plasma microRNA for early detection of gastric cancer. Mol Biol Rep 40: 2091-2096, 2013.

9. Kowal J, Tkach M and Théry C: Biogenesis and secretion of exosome. Curr Opin Cell Biol 29: 116-125, 2014.

10. Ge Q, Zhou Y, Lu J, Bai Y, Xie X and Lu Z: miRNA in plasma exosome is stable under different storage conditions. Molecules 19: 1567-1575, 2014.

11. Colombo M, Raposo G and Théry C: Biogenesis, secretion and intercellular interactions of exosomes and other extracellular vesicles. Ann Rev Cell Dev Biol 30: 255-289, 2014.

12. Kadota T, Yoshioka Y, Fujita Y, Kuwano K and Ochiya T: Extracellular vesicles in lung cancer-From bench to bedside. Semin Cell Dev Biol 67: 39-47, 2017.

13. Ogata-Kawata H, Izumiya M, Kurioka D, Honma Y, Yamada Y, Furuta K, Gunji T, Ohta H, Okamoto H, Sonoda H, et al: Circulating exosomal microRNAsas biomarkers of colon cancer. PLoS One 9: e92921, 2014.

14. Hannafon BN and Ding WQ: Intercelluar communication by exosome-derived microRNAs in cancer. Int J Mol Sci 14: 14240-14269, 2013.

15. Tovar-Camargo OA, Toden S and Goel A: Exosomal microRNA biomarkers: Emerging frontiers in colorectal and other human cancers. Expert Rev Mol Diagn 16: 553-567, 2016.

16. Dejima H, Iinuma H, Kanaoka R, Matsutani N and Kawamura K: Exosomal microRNA in plasma as a non-invasive biomarker for the recurrence of non-small cell lung cancer. Oncol Lett 13: 1256-1263, 2017.

17. Tsukamoto M, Iinuma H, Yagi T, Matsuda K and Hashiguchi Y: Circulating exosomal MicroRNA-21 as a biomarker in each tumor stage of colorectal cancer. Oncology 92: 360-370, 2017.

18. Japanese Gastric Cancer Association: Japanese gastric cancer treatment guidelines 2014 (ver.4). Gastric Cancer 20: 1-19, 2017. 
19. Salvi A, Sabelli C, Moncini S, Venturin M, Arici B, Riva P, Portolani N, Giulini SM, De Petro G and Barlati S: MicroRNA-23b mediates urokinase and c-met downmodulation and a decreased migration of human hepatocellular carcinoma cells. FEBS J 276: 2966-2982, 2009.

20. Li W, Liu Z, Chen L, Zhou L and Yao Y: MicroRNA-23b is an independent prognostic marker and suppresses ovarian cancer progression by targeting runt-related transcription factor- 2 . FEBS Lett 588: 1608-1615, 2014.

21. Loftus JC, Ross JT, Paquette KM, Paulino VM, Nasser S, Yang Z, Kloss J, Kim S, Berens ME and Tran NJ: miRNA expression profiling in migration glioblastoma cells: Regulation of cell migration and invasion by miR-23b via targeting of Pyk2. PLoS One 7: e39818, 2012.

22. Zhang H, Hao Y, Yang J, Zhou Y, Li J, Yin S, Sun C, Ma M, Huang $\mathrm{Y}$ and $\mathrm{Xi} \mathrm{JJ}$ : Genome-wide functional screening of $\mathrm{miR}-23 \mathrm{~b}$ as a pleiotropic modulator suppressing cancer metastasis. Nat Commun 2: 554, 2011.

23. Pellegrino L, Stebbing J, Braga VM, Frampton AE, Jacob J Buluwela L, Jiao LR, Periyasamy M, Madsen CD, Caley MP, et al: miR-23b regulates cytoskeletal remodeling, motility and metastasis by directly targeting multiple transcripts. Nucleic Acids Res 41: 5400-5412, 2013.

24. Majid S, Dar AA, Saini S, Arora S, Shahryari V, Zaman MS, Chang I, Yamamura S, Tanaka Y, Deng G and Dahiya R: miR-23b represses proto-oncogene Src kinase and functions as methylation-silenced tumor suppressor with diagnostic and prognostic significance in prostate cancer. Cancer Res 72: 6435-6446, 2012.

25. Goto Y, Kojima S, Nishikawa R, Enokida H, Chiyomaru T, Kinoshita T, Nakagawa M, Naya Y, Ichikawa T and Seki N: The microRNA-23b/27b/24-1 cluster is a disease progression marker and tumor suppressor in prostate cancer. Oncotarget 5: $7748-7759,2014$
26. Kou CH, Zhou T, Han XL, Zhuang HJ and Qian HX: Downregulation of miR-23b in plasma is associated with poor prognosis in patients with colorectal cancer. Oncol Lett 12: 4838-4844, 2016

27. Ma G, Dai W, Sang A, Yang X and Gao C: Upregulation of microRNA-23a/b promotes tumor progression and confers poor prognosis in patients with gastric cancer. Int J Clin Exp Pathol 7: 8833-8840, 2014

28. Sakuramoto S, Sasako M, Yamaguchi T, Kinoshita T, Fujii M, Nashimoto A, Furukawa H, Nakajima T, Ohashi Y, Imamura $\mathrm{H}$, et al: Adjuvant chemotherapy for gastric cancer with S-1, an oral fluoropyrimidine. N Engl J Med 357: 1810-1820, 2007.

29. Sasako M, Sakuramoto S, Katai H, Kinoshita T, Furukawa H, Yamaguchi T, Nashimoto A, Fujii M, Nakajima T and Ohashi Y: Five-year outcomes of a randomized phase III trial comparing adjuvant chemotherapy with S-1 versus surgery alone in stage II or III gastric cancer. J Clin Oncol 29: 4387-4393, 2011.

30. Bang YJ, Van Cutsem E, Feyereislova A, Chung HC, Shen L, Sawaki A, Lordick F, Ohtsu A, Omuro Y, Satoh T, et al: Trastuzumab in combination with chemotherapy versus chemotherapy alone for treatment of HER2-positive advanced gastric or gastro-oesophageal junction cancer (ToGA): A phase 3 , open-label, randomised controlled trial. Lancet 376: 687-697, 2010.

31. An Y, Zhang Z, Shang Y, Jiang X, Dong J, Yu P, Nie Y and Zhao Q: miR-23b-3p regulates the chemoresistance of gastric cancer cells by targeting ATG12 and HMGB2. Cell Death Dis 6: e1766, 2015.

32. Zaman MS, Thamminana S, Shahryari V, Chiyomaru T, Deng G, Saini S, Majid S, Fukuhara S, Chang I, Arora S, et al: Inhibition of PTEN gene expression by oncogenic miR-23b-3p in renal cancer. PLoS One 7: e50203, 2012 